\title{
Comparative Ultrasound Review of Free Intra-Peritoneal Fluid (Ascites)
}

\section{Adam Moyosore Afodun1,2*, Ejike Daniel Eze ${ }^{3}$, Airat Adeola Bakare4, Victor Okoliko Ukwenya ${ }^{5}$, Taofeek Olarewaju Ayinde6, Khadijah Kofoworola Quadri' ${ }^{6}$}

\author{
${ }^{1}$ Department of Anatomy, Faculty of Biomedical Sciences, Kampala International University, Kampala, Uganda \\ ${ }^{2}$ Department of Radiology, Ultrasound and Doppler Unit, Crystal Specialist Hospital, Lagos, Nigeria \\ ${ }^{3}$ Department of Physiology, Faculty of Biomedical Sciences, Kampala International University, Kampala, Uganda \\ ${ }^{4}$ Department of Anatomy, College of Medicine, University of Lagos, Lagos, Nigeria \\ ${ }^{5}$ Department of Anatomy, Federal University of Technology Akure, Akure, Nigeria \\ ${ }^{6}$ Department of Physiology, Faculty of Basic Medical Sciences, University of Ilorin, Ilorin, Nigeria \\ Email: *afodunadam@yahoo.com
}

\begin{abstract}
How to cite this paper: Afodun, A.M., Eze, E.D., Bakare, A.A., Ukwenya, V.O., Ayinde, T.O. and Quadri, K.K. (2017) Comparative Ultrasound Review of Free Intra-Peritoneal Fluid (Ascites). Open Journal of Medical Imaging, 7, 229-236.

https://doi.org/10.4236/ojmi.2017.74021
\end{abstract}

Received: October 1, 2017

Accepted: November 21, 2017

Published: November 24, 2017

Copyright (c) 2017 by authors and Scientific Research Publishing Inc. This work is licensed under the Creative Commons Attribution International License (CC BY 4.0).

http://creativecommons.org/licenses/by/4.0/

\begin{abstract}
The aim of this study is to review clinical case ascites and the use of ultrasound for the detection/classification of free intra-peritoneal fluid. Current therapeutic modality is in support of ultrasound guided paracentesis/thoracocentesis as a multidisciplinary procedure for ascitic reduction. Post-surgical free intra-peritoneal fluid is a common iatrogenic complication. We present ascitic cases of diseased conditions, questionable origins (in females) following hernia reduction and laparoscopic procedures to evaluate ascitic etiology through ultrasound. Average attenuation and quadrant sum of largest fluid pocket ultrasound (frozen) images were measured. 12 patients with free intra-peritoneal fluid had identifiable anatomical organs: liver, kidneys, urinary bladder and bowel loops. The size of maximal pocket of ascitic fluid in ill-patients was $1210 / 300 \mathrm{~mm}^{2}$, while fluid in subjects with non-traumatic injury was clear (free of striations) and appeared less dense.
\end{abstract}

\section{Keywords}

Ascites, Fluid, Ultrasound, Injury

\section{Introduction}

In quantifying the volume of ascites in evaluating disease extent and selecting appropriate means of treatment, ultrasound use is important because physical palpation and history taking are less sensitive with accuracy [1]. The purpose of this review is to determine ascitic free-fluid volume in female patients with dif- 
ferent underlying ailments and pathology. For a recommended diagnostic paracentesis greater than $20 \mathrm{ml}$ of fluid must be presented [2]; other workers [3] earlier stated a minimum of $80 \mathrm{cc}(80 \mathrm{ml})$ of fluid is needed.

Germ peritonitis (GP), a lethal infection with high mortality rate is a possible complication of ascites [4]. Abdominal bloating and distension with patients Roberts' Sign (gaseous ooze) produce a filling effect. Individual quadrant measurements of maximal fluid can be estimated in gauging ascites [5]. Literature reports greater than $52 \%$ of patients with hepatic disease and cirrhosis develop ascites [6] and 6\% "ascitic-link" for heart diseases and cardio-pulmonary failure. A poly-morpho-nuclear (PMN) leukocyte count $\geq 250$ cells $/ \mathrm{mm}^{3}$ in ascitic condition is diagnostic of Spontaneous Bacterial Peritonitis (SBP) [7]. Greater than $>95 \mathrm{ml}$ is considered as a gold standard for the diagnosis of ascites [8]. Extracted ascitic fluid volume recommended for histopathological analysis will depend on the (explorative, therapeutic or diagnostic) nature/indication of the procedure.

However, when volumetric comparison was made between the 2 groups (Diseased condition/without identifiable injury and those with blunt trauma) significant differences were observed between images $P<0.0001$. This means some pathological diseases may have smaller quantity of ascitic fluid volume compared to patients with ballistic injuries.

\section{Materials and Methods of Case Reports}

All ultrasound was performed at Crystal Specialist Hospital, Dopemu-Akowonjo Lagos, with a General Electric (GE) Pro-Logic-3 ultrasound machine (made in the USA) between the months of August 2015 and November 2016. Patients voluntarily participated with obtained informed consent in line with the 1975 Helsinki Declaration on patient rights. Demographic data of patients were women between the age of 25 and 45 years, minimum of high school qualification, religious identity; Muslims 5, Christians 6 and African traditional religion 1. All were of average socio-economic status, 4 patients admitted for observation was based solely on the presence of free fluid. Patients' (quetelet) Body Mass Index (BMI) varied between $18.5-26 \mathrm{~kg} / \mathrm{m}^{2}$.

Ascitic severity was placed on a four-point scale D, C, B, A (see Figures 1-4) [8].

- Grade IV (D) Acute/Chronic range (>1200 - 1900) ml

- Grade III (C) Massive/severe TEAA range (>900 - 1200) ml

- Grade II (B) Moderate TEAA range (>700 - 900) ml

- Grade I (A) Mild TEAA range (>500 - 700) ml

To localize and isolate ascites in our patients, 2 methods were used namely; ultrasound and physical palpation by a physician.

During ultrasonography, the methodology used is similar to (liquor volume) amniotic fluid quantification, which required "frozen" images in longitudinal and traverse planes with largest ascitic pocket or pool. 3D $(\mathrm{L} \times \mathrm{H} \times \mathrm{W})$ and 


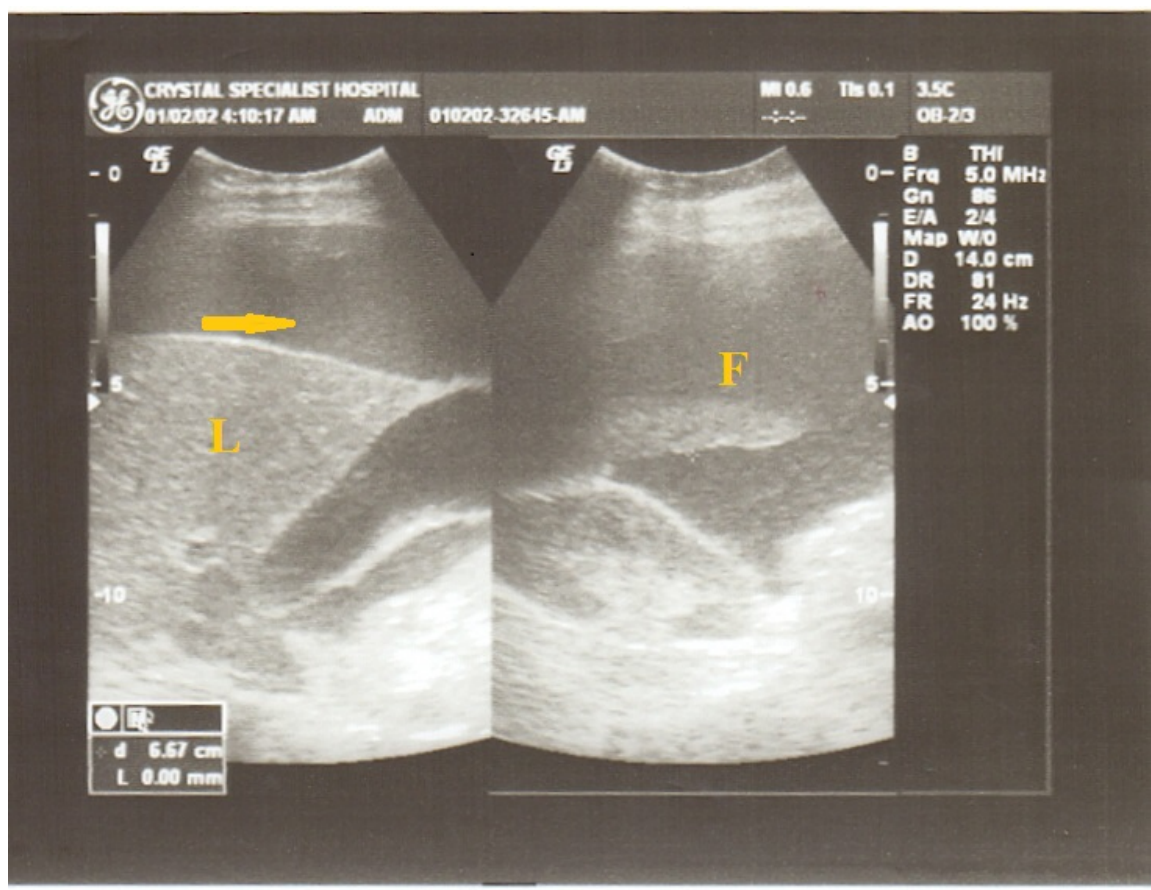

Figure 1. Image shows large amount of free-fluid (arrows) in the intra-epigastric region near the liver (L). Largest pocket of ascitic fluid (F) measured $1298 \mathrm{~mm}^{3}$ in volume. Patient underwent a conservative paracentesis which was uneventful after follow-up. Special consideration should be given to varying dimensions of gall bladder and liver due to atypical anatomy. Though ascitic enveloping provides better sonar penetration, general viscera appear smaller, with fluid volume averaging $1600 \mathrm{ml}$.

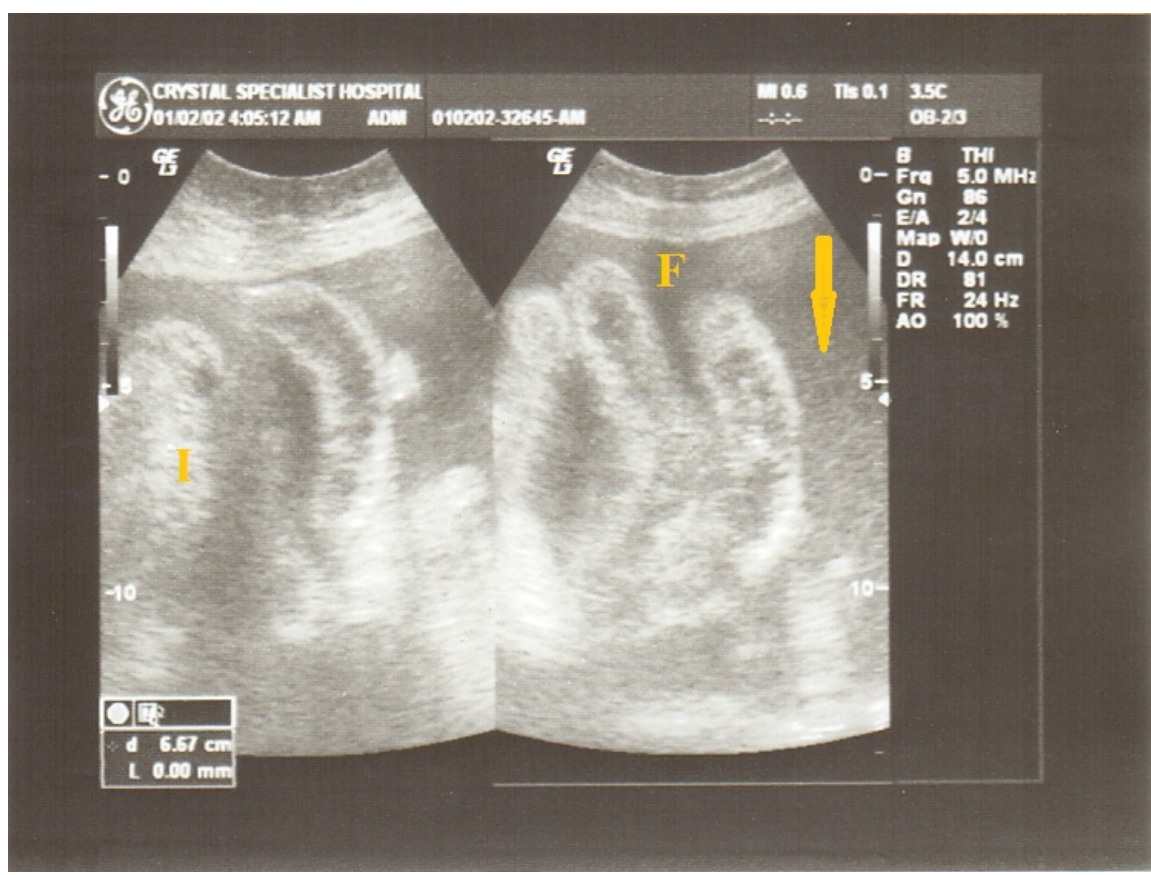

Figure 2. Free ascitic fluid within paracolic gutters with largest quadrant-pocket of free-fluid measured at $> \pm 130 \mathrm{~mm}$ in length (L). Note sonic evidence of intestinal (I) thickening. Floating bowel loops in the right upper quadrant (RUQ) at the jejuno-illeal region; free intraperitoneal fluid averaging $1100 \mathrm{ml}$. 


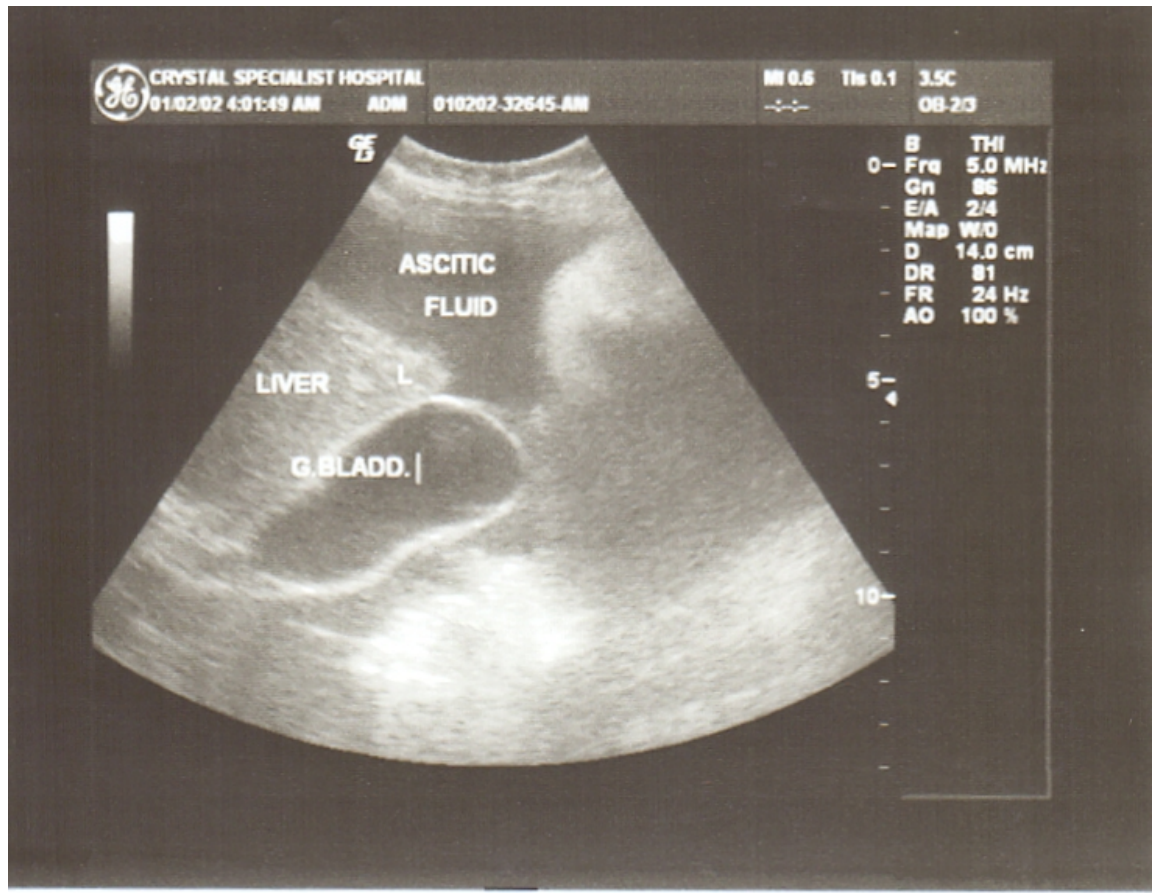

Figure 3. Observation for ascitic "free-zone", the ascites filled space proximal to the hepato-renal angle. No fibrin strands or edematous abdominal wall seen in the peritoneum. Before therapeutic paracentesis (ascitic-safe-zones above the intestine and mesentery; below abdominal wall for safe syringe placement), some physicians prefer right paracolic gutter approach. Ascitic volume averages $800 \mathrm{ml}$.

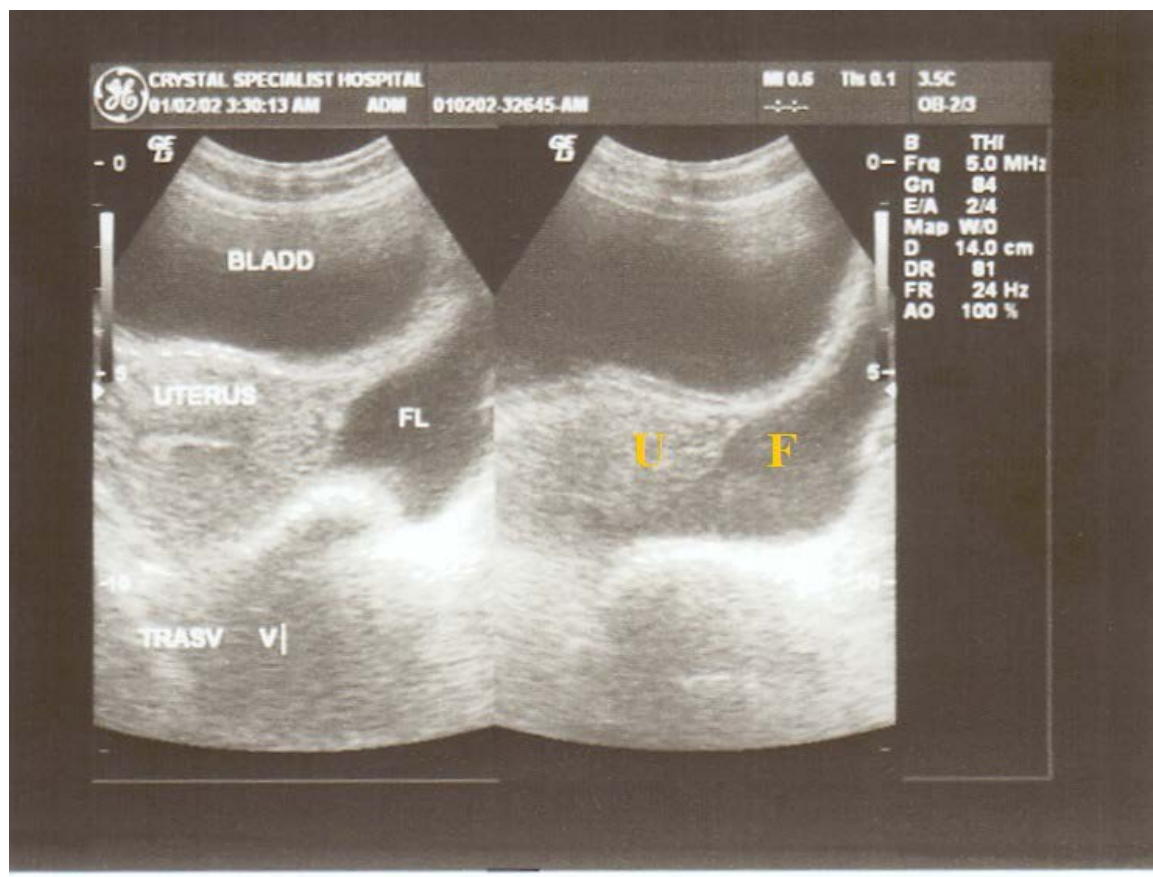

Figure 4. Ultrasound of ascites (F) with curvilinear probe/transducer. Note the acute Pelvic Inflammatory Disease (PID) enveloping the uterus (U), cul-de-sac (Douglas pouch) and (acute) in the left adnexal area. In addition, impending genesis of bilateral hydro/pyosalphinx; fluid volume average $=600 \mathrm{ml}$. 
2D $(\mathrm{L} \times \mathrm{B})$. Total Estimated Abdominal Ascites (TEAA) is measured by average volumetry of quadrant pools; i.e. $\left(\mathrm{V}_{1}\right.$ pool $)+\mathrm{V}_{2}+\mathrm{V}_{3}+\mathrm{V}_{4}$ similar to the methodology of Szkodziak et al., (2010) [1].

\section{Sonogram Analysis}

Sonologists and Anatomists with over 10 years experience performed a joint review of the ultrasound images. Determination was made on (blunt/pathologic), non-traumatic or traumatic genesis of the free-fluid. Pathological cause of ascites was noticed from the medical records and history of patients. It is however required to rule-out other causes of ascites. The supra-pelvic region in females was proximally examined since it is a region were ascites is frequently seen (Figure 4). Back-up technique was implemented using the Smallest Fluid Depth (SFD) i.e. the distance from the "highest" colic tissue (intestine) to the abdominal wall.

\section{Discussion}

Accurate knowledge of surface anatomy, safety standards and infectious complications are some of the limitations associated with ultrasound guided paracentesis procedure [9] [10]. Figure 3 and Figure 4 are in agreement with Young-Cheong et al., 2017 [11]; David et al., 2017 [12] and Evan et al., 2017 [13]; as unexplained haemo-peritoneum occurs mostly from intestinal injury, mesenteric-shunt or colic inflammation.

Therefore, ascitic quantification will aid internal physicians and general surgeons in determining the significance of isolated ascites. Though earlier reported in literature, less than $<7 \%$ of patients with isolated free-fluid have occult bowel injury as noted by Livington et al., (2001) [14].

In agreement with Yamada et al., (2003) [15] the priority to investigate the cause of ascites will determine its management. Standard medical procedure supports the use of sonar prior to puncture procedure (paracentesis) to avoid iatrogenic complications. A longitudinal study [16] that compared blind paracentesis to ultrasound guided procedure elaborated on fluid aspiration rate. Estimated amount of ascitic fluid is mostly prone to difficulty in obtaining accurate measurements through "frozen" image attenuation (in small pockets) which is often encountered. Sonogram acquisition of free intraperitoneal fluid views suggest significant volume comparison were statistically significant. 12 patients with free intra-peritoneal fluid had identifiable anatomical organs: liver, kidneys, urinary bladder and bowel loops. The size of maximal pocket of ascitic fluid in ill-patients was 1210/300 $\mathrm{mm}^{2}$ (Figure 1 and Figure 3), while fluid in subjects with non-traumatic injury was clear (free of striations) and appeared less dense (Figure 2). The patients showed lower rate of complications on ultrasound unlike what was observed previously with patients exhibiting generalized peritonitis. For patients with ascites and identifiable pathology, no significant distinction $(p=0.87)$ was found in the sonographic delayed phase images and "frozen" attenuation dimensions. 
The peritoneal cavity may be subject to inflammatory reactions from surgery, parcentesis or laparoscopic exposure in cases were visceral organs had no known prior allergy [17] [18] [19]. Taking clinical etiology of ascites into consideration with sonogram analysis, frequency of iatrogenic laparotomy may be avoided. Difference in mean attenuation between trauma causing ascites and pathologic ascites was statistically significant; parallel to the assertion of Victor et al., (2015) [20]; Hasan et al., (2015) [21]; Laura et al., (2014) [22]; Michael et al., (2014) [23] and Wei-Chon et al., (2014) [24] [25]. When no definitive causative factor is the source of peritoneal fluid accumulation, bowel/urinary tract injury should be excluded. Focused attention needs to be paid to intestines (Figure 2) and visceral organs as hospital records may sometimes be incomplete, a variable we cannot control.

\section{Limitations}

A major limitation is that grey scale ultrasound cannot distinguish between various types of body fluids (pus-exudate, blood, ascites, mucous etc). We believe the texture, density and properties of a fluid with respect to adjourning abdominal organs are important. However, this is where clinical knowledge is applied. There are no universally accepted way of ascitic fluid quantification, still debated amongst Sonologists and Internal Physicians. Patients are sometimes unreliable in keeping radiological appointments for scan (and ascitic quantification), thereby occasionally warranting estimates. It should be noted that real time ultrasound guided paracentesis (ascites drainage) is susceptible to a major complication; danger of hemorrhage into the peritoneal cavity.

\section{Conclusions}

Results revealed the most common causes of ascites in Nigerian patients were Pelvic Inflammatory Disease (PID), hepatitis, abdominal tuberculosis (TB), renal diseases, urinary bladder schistosomiasis, poly-cystic ovarian syndrome (PCOS), ovarian carcinoma, liver cirrhosis and cardiac ailments. Since ultrasound is non-invasive, economical and reliably differentiates between exudates and transudates by measuring the ascitic fluid volume, it is recommended to be the foremost imaging diagnostic modality for the above conditions. Physicians caring for critical bed-side patients should employ sonography as an indispensable tool to help facilitate a peritoneal-tap or paracentesis procedure as the case may apply.

Further studies incorporating Color Doppler ultrasonography (as opposed to monochrome grey-scale) should be recommended to exclude vascular leakage into ascitic pool.

\section{References}

[1] Szkodziak, P.R., Wozniak, S., Czuczwar, P., et al. (2010) Ascites Index-A New Method of Ultrasound Evaluation of Ascites Volume in Patients with Ovarian Can- 
cer. Ultrasound in Obstetrics and Gyneacology, 36, 289. https://doi.org/10.1002/uog.8732

[2] World Gastroenterology Organisation (WGO) (2014) Practice Guideline: Condition; Management of Ascites Complicating Cirrhosis in Adults.

http://www.worldgastroenterology.org/guidelines/global-guidelines

[3] Bard, C., Lafortune, M. and Breton G. (1986) Ascites: Ultrasound Guidance or Blind Paracentesis? Canadian Medical Association Journal, 135, 209-210.

[4] Chinasck, B., Afarian, H., Minnigan, H., et al. (2008) Physician Clinical Impression Does not Rule out Spontaneous Bacterial Peritonitis in Patients Undergoing Emergency Department Paracentesis. Annals of Emergency Medicine, 52, 268-273. https://doi.org/10.1016/j.annemergmed.2008.02.016

[5] Irshad, A., Ackerman, S., Anig, M. et al. (2009) Can the Smallest Depth of Ascitic Fluid on Sonograms Predict the Amount of Drainable Fluid? Journal of Clinical UItrasound, 37, 440-444. https://doi.org/10.1002/jcu.20616

[6] Krige, J.E. and Beckingham, I.J. (2001) ABC of Diseases of Liver, Pancreases, and Billiary System: Portal Hypertension-2. Ascites, Encephalopathy, and Other Conditions. BMJ, 322, 416-418. https://doi.org/10.1136/bmj.322.7283.416

[7] McCribbon, A., Chen, G., Peltekian, K., et al. (2007) An Evidence-Based Manual for Abdominal Paracentesis. Digestive Diseases and Sciences, 52, 3307-3315.

https://doi.org/10.1007/s10620-007-9805-5

[8] Alnumeiri, M.S., Ayad, C.E., Ahmed, B.H. and Abdalla, E.A. (2015) Evaluation of Ascites and Its Etiology Using Ultrasonography. Journal of Research and Development, 3, 119.

https://www.omicsonline.org/open-access/evaluation-of-ascites-and-its-etiology-usi ng-ultrasonography-jrd-119.pdf

[9] Webster, S.T., Brown, K.L., Lucey, M.R., et al. (1996) Hemorrhagic Complications of Large Volume Paracentesis. The American Journal of Gastroenterology, 91, 366-368.

[10] Grabau, C.M., Crago, S.F., Hoff, L.K., et al. (2004) Performance Standards for the Therapeutic Abdominal Paracentesis. Hepatology, 40, 484-488. https://doi.org/10.1002/hep.20317

[11] Wong, Y.-C., Wang, L.-J., Wu, C.-H. and Hsu, Y.-P. (2017) Peritoneal Fluid of Low CT Hounsfield Units as a Screening Criterion for Traumatic Bowel Perforation. Japanese Journal of Radiology, 35, 145-150. https://doi.org/10.1007/s11604-017-0613-3

[12] Bates D.D.B., Wasserman M., Malek A., et al. (2017) Multi Detection CT of Surgically Proven Blunt Bowel and Mesenteric Injury. Radio Graphics, 37, 613-625. https://doi.org/10.1148/rg.2017160092

[13] Jost E., Roberts, D.J. and Penney, T. (2017) Accuracy of Clinical, Laboratory, and Computed Tomography Findings for Identifying Hollow Victims in Blunt Trauma Patients with Unexplained Intraperitoneal Free Fluid without Solid Organ Injury. The American Journal of Surgery, 213, 874-880. https://doi.org/10.1016/j.amjsurg.2017.03.016

[14] Livington, D.H., lavery, R.F., Passannante, M.R., et al. (2001) Free Fluid on Abdominal Computed Tomography without Solid Organ Injury after Blunt Abdominal Injury Does Not Mandate Celiotomy. The American Journal of Surgery, 182, 6-9. https://doi.org/10.1016/S0002-9610(01)00665-1

[15] Yamada, T., Alpers, D.H., Owyang, C., et al. (2008) Principles of Clinical Gastroen- 
terology. Blackwell Publishing, Hoboken, New Jersey. https://doi.org/10.1002/9781444300758

[16] Nazeer, S.R., Dewbre, H. and Miller, A.H. (2005) Ultrasound-Assisted Paracentesis Performed by Emergency Physicians vs. the Traditional Technique: A Prospective, Randomized Study. American Journal of Emergency Medicine, 23, 363-367. https://doi.org/10.1016/j.ajem.2004.11.001

[17] Milingos, S., Protopagas, A., Chatzipapas, I., et al. (2001) Postoperative Ascites Developing after Laparoscopic Surgery Can Become a Difficult Diagnostic Dilemma. Journal of the American Association of Gynecologic Laparoscopists, 8, 587-590. https://doi.org/10.1016/S1074-3804(05)60626-3

[18] Zhao, X., Wang, M., Huang, H. and Wang, X. (2005) Idiopathic Postoperative Ascites after Laparoscopic Salpingectomy for Ectopic Pregnancy. Journal of Minimally Invasive Gynecology, 12, 439-441. https://doi.org/10.1016/j.jmig.2005.04.005

[19] Hupuczi, P. and Papp, Z. (2005) Postoperative Ascites Associated with Intraperitoneal Antiseptic Lavage. Obstetrics and Gynecology, 105, 1267-1268. https://doi.org/10.1097/01.AOG.0000159552.19168.cd

[20] Kong, V.Y., Jeetoo, D. and Clarke, D.L. (2015) Isolated Free Intra-Abdominal Fluid on CT in Blunt Trauma: The Continued Diagnostic Dilemma. Chinese Journal of Radiology and Nuclear Medicine, 46, 1-8.

[21] Hasan, I., Gehan, S.S. and Hussien, A.E. (2015) The Diagnostic Role of MDCT Enterography in Small Bowel Lesions. The Egyptian Journal of Radiology and Nuclear Medicine, 46, 1-8. https://doi.org/10.1016/j.ejrnm.2014.09.006

[22] Laura, N., Stephen, C.H. and Krista, L.K. (2014) Isolated Free Fluid on Abdominal Computed Tomography in Blunt Trauma. Watch and Wait or Operate? Journal of the American College of Surgeons, 219, 599-605. https://doi.org/10.1016/j.jamcollsurg.2014.04.020

[23] Michael, G.F., Jefferson, I.B., Tausha, S., et al. (2014) Isolated Pelvic Fluid in Males on Outpatient Magnetic Resonance Imaging Examinations. Journal of Computer Assisted Tomography, 1.

[24] Wei-chon, C., Kai-Hsiung, K., Chunshu, L., et al. (2014) Features on MDCT That Predict Surgery in Patients with Adhesive-Related Small Bowel Obstruction. DLOS ONE, 9, $2 \mathrm{e} 89804$.

[25] Ennis, J., et al. (2014) Ultrasound for Detection of Ascites and for Guidance of the Paracentesis Procedure: Technique and Review of the Literature. International Journal of Clinical Medicine, 5, 1277-1293. https://doi.org/10.4236/ijcm.2014.520163 